MATHEMATICS OF COMPUTATION

Volume 75, Number 255, July 2006, Pages 1201-1215

S 0025-5718(06)01844-8

Article electronically published on May 3, 2006

\title{
THE STABILITY OF MODIFIED RUNGE-KUTTA METHODS FOR THE PANTOGRAPH EQUATION
}

\author{
M. Z. LIU, Z. W. YANG, AND Y. XU
}

\begin{abstract}
In the present paper, the modified Runge-Kutta method is constructed, and it is proved that the modified Runge-Kutta method preserves the order of accuracy of the original one. The necessary and sufficient conditions under which the modified Runge-Kutta methods with the variable mesh are asymptotically stable are given. As a result, the $\theta$-methods with $\frac{1}{2} \leq \theta \leq 1$, the odd stage Gauss-Legendre methods and the even stage Lobatto IIIA and IIIB methods are asymptotically stable. Some experiments are given.
\end{abstract}

\section{INTRODUCTION}

In this paper we consider the pantograph equation

$$
\left\{\begin{array}{l}
x^{\prime}(t)=f(t, x(t), x(q t)), \quad t \geq 0, \\
x(0)=x_{0}
\end{array}\right.
$$

where $0<q<1, x_{0} \in \mathbb{C}$ and $f: \mathbb{R}^{+} \times \mathbb{C} \times \mathbb{C} \rightarrow \mathbb{C}$ is continuous.

This system can be found in modelling many phenomena such as, for example, electrodynamics [7, 18, nonlinear dynamical systems [5, and so on. For a comprehensive list see 9. Some results about the analytic solutions have been shown in 4, 9, 11, 13.

There have been a lot of papers concerning the numerical stability of the test equation

$$
\left\{\begin{array}{l}
x^{\prime}(t)=a x(t)+b x(q t), \quad t \geq 0, \\
x(0)=x_{0},
\end{array}\right.
$$

where $a, b, x_{0}$ are complex constants and $q \in(0,1)$. The most difficult problem is the limited computer memory as shown in 16, 17. There are two ways to avoid the storage problem. One way is by transforming (1.2) to an equation with constant time lag and variable coefficients as in [14, 15,

$$
\begin{cases}x^{\prime}(t)=a e^{t} x(t)+b e^{t} x(t+\log q), & t \geq t_{0}, \\ x(t)=x_{0}(t), & t_{0}+\log q \leq t \leq t_{0},\end{cases}
$$

where $x_{0}(t)$ is a continuous function in $\left[t_{0}+\log q, t_{0}\right]$, and by applying the numerical methods with a constant mesh.

Received by the editor September 13, 2004.

2000 Mathematics Subject Classification. Primary 65L02, 65L05; Secondary 65L20.

Key words and phrases. Pantograph equation, asymptotical stability, Runge-Kutta methods.

This paper was supported by the National Natural Science Foundation of China (10271036).

(C)2006 American Mathematical Society 
The other way is by applying numerical methods with the quasi-geometric mesh to (1.2), directly, as in [2, 3, [16, 17, 19]. Other reason for applying numerical methods with the quasi-geometric mesh is that, when using the numerical methods with constant mesh, we obtain a difference system not of fixed order whose analysis is significantly more difficult, as shown in [10, 12.

From [19, we can see that the $\theta$-methods with $\theta=\frac{1}{2}$, the Gauss-Legendre methods and the Lobatto IIIA methods are not asymptotically stable. Until now there have been few papers which concern the stability of the Lobatto IIIB methods for the pantograph equation.

In the present paper, we construct a modified Runge-Kutta method and show that the method preserves the order of accuracy of the original one. We also give the necessary and sufficient conditions for the asymptotical stability of the modified Runge-Kutta method for (1.1) and lastly some experiments.

\section{Runge-Kutta methods}

In this section we will construct the modified Runge-Kutta method and show that this method preserves the order of accuracy of the original one.

Consider

$$
\begin{aligned}
& y^{\prime}=f(t, y), \quad 0 \leq t \leq T, \\
& y(0)=y_{0},
\end{aligned}
$$

where $y_{0} \in \mathbb{C}, T>0$ and $f: \mathbb{R}^{+} \times \mathbb{C} \rightarrow \mathbb{C}$ is a continuous function.

Let $\Delta=\left\{0=t_{0}<t_{1}<\cdots<t_{n}=T\right\}$ be a mesh and $h_{n+1}=t_{n+1}-t_{n}$. The modified Runge-Kutta method is defined by

$$
\begin{aligned}
& \bar{y}_{n+1}=\bar{y}_{n}+h_{n+1} \sum_{i=1}^{s} b_{i} f\left(t_{n}+c_{i} h_{n+1}, \bar{Y}_{i}^{n+1}\right), \\
& \bar{Y}_{i}^{n+1}=\bar{y}_{n}+\bar{h}_{n+1} \sum_{j=1}^{s} a_{i j} f\left(t_{n}+c_{j} h_{n+1}, \bar{Y}_{j}^{n+1}\right), \quad i=1,2, \ldots, s,
\end{aligned}
$$

and $\bar{h}_{n+1}=\left(1+\alpha_{n+1}\left(h_{n+1}\right)\right) h_{n+1}$ with $\alpha_{n+1}(\eta)$ such that

(H1) $\alpha_{n+1}(\eta)=O\left(\eta^{\bar{p}}\right)$ as $\eta \rightarrow 0$ for all $n \geq 0$.

Theorem 2.1. Suppose that the Runge-Kutta method $(A, b, c)$ is of order $p$ and $f(t, y)$ is of class $C^{p}$. Then the order of the Runge-Kutta method (2.2), (2.3) is $p^{\prime}=\min \{p, \bar{p}+1\}$.

Proof. Let $y_{n+1}$ and $Y^{n+1}=\left(Y_{1}^{n+1}, Y_{2}^{n+1}, \ldots, Y_{s}^{n+1}\right)^{T}$ be the solutions of the $p$ order Runge-Kutta method, i.e.,

$$
\begin{aligned}
& y_{n+1}=y_{n}+h_{n+1} \sum_{i=1}^{s} b_{i} f\left(t_{n}+c_{i} h_{n+1}, Y_{i}^{n+1}\right), \\
& Y_{i}^{n+1}=y_{n}+h_{n+1} \sum_{j=1}^{s} a_{i j} f\left(t_{n}+c_{j} h_{n+1}, Y_{j}^{n+1}\right), \quad i=1,2, \ldots, s,
\end{aligned}
$$

and $\bar{y}_{n}=y_{n}=y\left(t_{n}\right)$. Then it is sufficient to prove

$$
\left|\bar{y}_{n+1}-y_{n+1}\right|=O\left(h_{n+1}^{p^{\prime}+1}\right) \text { as } h_{n+1} \rightarrow 0,
$$

since $\left|y_{n+1}-y\left(t_{n+1}\right)\right|=O\left(h_{n+1}^{p+1}\right)$ as $h_{n+1} \rightarrow 0$. 
In view of (2.3) and (2.5) we can obtain

$$
\begin{aligned}
\bar{Y}_{i}^{n+1} & -Y_{i}^{n+1} \\
= & \bar{h}_{n+1} \sum_{j=1}^{s} a_{i j} f\left(t_{n}+c_{j} h_{n+1}, \bar{Y}_{j}^{n+1}\right)-h_{n+1} \sum_{j=1}^{s} a_{i j} f\left(t_{n}+c_{j} h_{n+1}, Y_{j}^{n+1}\right) \\
= & h_{n+1} \alpha_{n+1}\left(h_{n+1}\right) \sum_{j=1}^{s} a_{i j} f\left(t_{n}+c_{j} h_{n+1}, \bar{Y}_{j}^{n+1}\right) \\
& +h_{n+1} \sum_{j=1}^{s} a_{i j}\left(f\left(t_{n}+c_{j} h_{n+1}, \bar{Y}_{j}^{n+1}\right)-f\left(t_{n}+c_{j} h_{n+1}, Y_{j}^{n+1}\right)\right) \\
= & h_{n+1} \alpha_{n+1}\left(h_{n+1}\right) \sum_{j=1}^{s} a_{i j} f_{j}+h_{n+1} \sum_{j=1}^{s} a_{i j}\left(f_{y}\right)_{j}\left(\bar{Y}_{j}^{n+1}-Y_{j}^{n+1}\right),
\end{aligned}
$$

where $f_{j}=f\left(t_{n}+c_{j} h_{n+1}, \bar{Y}_{j}^{n+1}\right),\left(f_{y}\right)_{j}=\frac{\partial f}{\partial y}\left(t_{n}+c_{j} h_{n+1}, \bar{Y}_{j}^{n+1}+\sigma_{j}\left(Y_{j}^{n+1}-\bar{Y}_{j}^{n+1}\right)\right)$, $0<\sigma_{i}<1, i=1,2, \ldots, s$. Consequently let $Z^{n+1}=\left(\bar{Y}_{1}^{n+1}-Y_{1}^{n+1}, \ldots, \bar{Y}_{s}^{n+1}-\right.$ $\left.Y_{s}^{n+1}\right)^{T}, F^{n+1}=\left(f_{1}, \ldots, f_{s}\right)^{T}$ and $F_{y}^{n+1}=\operatorname{diag}\left(\left(f_{y}\right)_{1}, \ldots,\left(f_{y}\right)_{s}\right)^{T}$, and we have

$$
Z^{n+1}=h_{n+1} \alpha_{n+1}\left(h_{n+1}\right) A F^{n+1}+h_{n+1} A F_{y}^{n+1} Z^{n+1} .
$$

Hence for sufficiently small $h_{n+1}, I-h_{n+1} A F_{y}^{n+1}$ is invertible and

$$
Z^{n+1}=h_{n+1} \alpha_{n+1}\left(h_{n+1}\right)\left(I-h_{n+1} A F_{y}^{n+1}\right)^{-1} A F^{n+1},
$$

which from (H1) and the boundedness of $F^{n+1}$ and $F_{y}^{n+1}$ implies that $\left\|Z^{n+1}\right\|=$ $O\left(h_{n+1}^{\bar{p}+1}\right)$ for a norm $\|\cdot\|$ in $\mathbb{C}^{s}$.

Therefore it is obvious from (2.2) and (2.4) that

$$
\begin{aligned}
\left|\bar{y}_{n+1}-y_{n+1}\right| & =h_{n+1}\left|\sum_{i=1}^{s} b_{i}\left(f\left(t_{n}+c_{i} h_{n+1}, \bar{Y}_{i}^{n+1}\right)-f\left(t_{n}+c_{i} h_{n+1}, Y_{i}^{n+1}\right)\right)\right| \\
& =h_{n+1}\left|b^{T} F_{y}^{n+1} Z^{n+1}\right|=O\left(h_{n+1}^{\bar{p}+2}\right),
\end{aligned}
$$

i.e., (2.6) is true and the proof is complete.

In a way similar to [1, we can show that the Runge-Kutta method (2.2), (2.3) is of order $p^{\prime}$ for the pantograph equation (1.1) step by step. In the following we assume that $\bar{p} \geq p-1$, therefore $p^{\prime}=p$.

For the general pantograph equation (1.1), the modified Runge-Kutta method is defined by

$$
\left\{\begin{array}{r}
\bar{y}_{n+1}=\bar{y}_{n}+h_{n+1} \sum_{i=1}^{s} b_{i} f\left(t_{n}+c_{i} h_{n+1}, \bar{Y}_{i}^{n+1}, y^{h}\left(q\left(t_{n}+c_{i} h_{n+1}\right)\right)\right), \\
\bar{Y}_{i}^{n+1}=\bar{y}_{n}+\bar{h}_{n+1} \sum_{j=1}^{s} a_{i j} f\left(t_{n}+c_{j} h_{n+1}, \bar{Y}_{j}^{n+1}, y^{h}\left(q\left(t_{n}+c_{j} h_{n+1}\right)\right)\right), \\
i=1,2, \ldots, s,
\end{array}\right.
$$

where $y^{h}\left(q\left(t_{n}+c_{i} h_{n+1}\right)\right)$ is an approximation to $y\left(q\left(t_{n}+c_{i} h_{n+1}\right)\right)$.

Here, the mesh $H=\left\{m ; t_{0}, t_{1}, \ldots, t_{n}, \ldots\right\}$ is introduced as follows. Let $\gamma_{0}>0$ be given, $t_{0}=\gamma_{0}$ and $t_{m}=q^{-1} \gamma_{0}$. We choose $m-1$ grid points $t_{1}<\cdots<t_{m-1}$ in 
$\left(t_{0}, t_{m}\right)$ and define the other points by

$$
t_{k m+i}=q^{-k} t_{i}, \quad \text { for } k=-1,0,1, \ldots, i=0,1, \ldots, m-1 .
$$

It is easy to see that the grid points $t_{n}$ such that $q t_{n}=t_{n-m}$ for $n \geq 0$ and the stepsize $h_{n}$ satisfies

$$
q h_{n}=h_{n-m} \text { for } n \geq 1 \text { and } \lim _{n \rightarrow \infty} h_{n}=\infty .
$$

Furthermore, we are supposed to have the numerical solution available until $\gamma_{0}$.

Therefore the relation (2.7) can be represented by

$$
\left\{\begin{array}{r}
\bar{y}_{n+1}=\bar{y}_{n}+h_{n+1} \sum_{i=1}^{s} b_{i} f\left(t_{n}+c_{i} h_{n+1}, \bar{Y}_{i}^{n+1}, \bar{Y}_{i}^{n-m+1}\right), \\
\bar{Y}_{i}^{n+1}=\bar{y}_{n}+\bar{h}_{n+1} \sum_{j=1}^{s} a_{i j} f\left(t_{n}+c_{j} h_{n+1}, \bar{Y}_{j}^{n+1}, \bar{Y}_{j}^{n-m+1}\right), \\
i=1,2, \ldots, s .
\end{array}\right.
$$

\section{Preliminary Results}

In this section we investigate the asymptotical stability of the linear difference equations with variable coefficients

$$
\left\{\begin{array}{l}
x(n+1)=M(n) x(n) \\
x\left(n_{0}\right)=x_{0}
\end{array}\right.
$$

where $M(n)=\left(m_{i j}(n)\right)$ is a $d \times d$ complex matrix function. In the following we assume that a matrix norm $\|\cdot\|$ is subordinate to some vector norm $\|\cdot\|$ and there is a matrix $M$ such that $\lim _{n \rightarrow \infty} M(n)=M$.

Lemma 3.1. Suppose that the algebraic multiplicity of the eigenvalue $\xi$ of $M$ is 1 and the other eigenvalues have a module less than one. Then if $|\xi| \geq 1$, there is a matrix norm $\|\cdot\|_{M}$ such that $\|M\|_{M}=\rho(M)$. Moreover, $\left\|M^{k}\right\|_{M}=\|M\|_{M}^{k}$ for all $k \in \mathbb{N}$.

Proof. According to the Jordan Canonical theorem in 8, there is a nonsingular matrix $P$ such that

$$
P^{-1} M P=\left(\begin{array}{ll}
\xi & \\
& \bar{M}
\end{array}\right),
$$

where $\bar{M}$ is an upper triangle matrix and $\rho(\bar{M})<1$. Let $D_{\delta}=\operatorname{diag}\left(\delta, \delta^{2}, \ldots, \delta^{d-1}\right)$; then for sufficiently small $\delta$

$$
\left\|D_{\delta}^{-1} \bar{M} D_{\delta}\right\|_{1}<|\xi|
$$

We define a matrix norm

$$
\|M\|_{M}=\left\|\left(\begin{array}{ll}
1 & \\
& D_{\delta}
\end{array}\right)^{-1} P^{-1} M P\left(\begin{array}{ll}
1 & \\
& D_{\delta}
\end{array}\right)\right\|_{1} .
$$

It is easily seen that

$$
\|M\|_{M}=|\xi|=\rho(M)
$$

The last statement of the lemma can be obtain from $\rho^{k}(M)=\rho\left(M^{k}\right) \leq\left\|M^{k}\right\|_{M} \leq$ $\|M\|_{M}^{k}=\rho^{k}(M)$.

It is easy to prove the following lemma by induction. 
Lemma 3.2. For some $n_{0} \in \mathbb{N}$, we denote

$$
\begin{aligned}
& F(n)=M(n)-M, \quad n=0,1, \ldots, \\
& B\left(n_{0}+k\right)= \begin{cases}F\left(n_{0}\right), & k=0 \\
M B\left(n_{0}+k-1\right)+F\left(n_{0}+k\right)\left(M^{k}+B\left(n_{0}+k-1\right)\right), & k \geq 1 .\end{cases}
\end{aligned}
$$

Then for $k \geq 0$,

$$
\prod_{j=n_{0}}^{n_{0}+k} M(j)=M^{k+1}+B\left(n_{0}+k\right) .
$$

If, in addition, the conditions in Lemma 3.1 are satisfied, then for $k \geq 0$,

$$
\left\|B\left(n_{0}+k\right)\right\|_{M} \leq \prod_{j=n_{0}}^{n_{0}+k}\left(\|M\|_{M}+\|F(j)\|_{M}\right)-\|M\|_{M}^{k+1} .
$$

Theorem 3.3. Assume that the conditions in Lemma 3.1 are satisfied and that there is a norm $\|\cdot\|_{*}$ such that $\sum_{n=0}^{\infty}\|M(n)-M\|_{*}<\infty$. Then for any given $n_{0}$ and $x_{0}$, the solution $x(n)$ satisfies $x(n) \rightarrow 0$ as $n \rightarrow \infty$ if and only if $|\xi|<1$.

Proof. If $|\xi|<1$, then $\rho(M)<1$. Therefore from [6] we can obtain that for any given $n_{0}$ and $x_{0}, x(n) \rightarrow 0$ as $n \rightarrow \infty$.

Suppose that $|\xi| \geq 1$. According to Lemma 3.1, there is a norm $\|\cdot\|_{M}$ such that $|\xi|=\|M\|_{M}$ and $\left\|M^{k}\right\|_{M}=\|M\|_{M}^{k}$. Since all norms are equivalent (see [8]), $\sum_{n=0}^{\infty}\|M(n)-M\|_{*}<\infty$ is equivalent to $\sum_{n=0}^{\infty}\|M(n)-M\|_{M}<\infty$. We choose $n_{0} \in \mathbb{N}$ such that

$$
\exp \left(\frac{1}{\|M\|_{M}} \sum_{n=n_{0}}^{\infty}\|M(n)-M\|_{M}\right)<2 .
$$

In view of Lemma 3.1 and Lemma 3.2, we have

$$
\begin{aligned}
\left\|\prod_{j=n_{0}}^{n_{0}+k} M(j)\right\|_{M} & =\left\|M^{k+1}+B\left(n_{0}+k\right)\right\|_{M} \\
& \geq\|M\|_{M}^{k+1}-\left\|B\left(n_{0}+k\right)\right\|_{M} \\
& \geq\|M\|_{M}^{k+1}-\left(\prod_{j=n_{0}}^{n_{0}+k}\left(\|M\|_{M}+\|F(j)\|_{M}\right)-\|M\|_{M}^{k+1}\right) \\
& \geq 2-\exp \left(\frac{1}{\|M\|_{M}} \sum_{n=n_{0}}^{\infty}\|F(n)\|_{M}\right)>0,
\end{aligned}
$$

which implies that the theorem is complete.

\section{The STABILITY ANALYSIS}

In this section we will investigate the numerical stability of (2.7) by applying the pantograph equation

$$
y^{\prime}(t)=\lambda y(t)+\mu y(q t), \quad t>0,
$$

where $\lambda, \mu \in \mathbb{C}, 0<q<1$ and the initial condition is $y(0)=y_{0} \in \mathbb{C}$. 
It is well known that the solution of (4.1) tends to zero if

$$
\operatorname{Re}(\lambda)<0, \quad|\mu|<|\lambda|
$$

where $\operatorname{Re}(\lambda)$ is the real part of $\lambda$. In the remainder of this paper we assume that $\alpha_{n+1}(\eta)$ satisfies (H1) and

(H2) $\alpha_{n+1}(\eta)>0$ for all $\eta>0$ and $n \geq 0$;

(H3) for any mesh $H$, there exists an $\alpha(H)>0$ such that $\left|\alpha_{n}\left(h_{n}\right)-\alpha(H)\right| \leq$ $q\left|\alpha_{n-m}\left(h_{n-m}\right)-\alpha(H)\right|$

(H4) $\inf _{H} \alpha(H)=0$.

Definition 4.1. Method (2.7) is called $H_{\alpha}$-stable if any application of (2.9) to (4.1) generates numerical approximations $\bar{y}_{n}$ which tend to zero as $n \rightarrow \infty$, for any $q$ with $0<q<1, H=\left\{m ; t_{0}, t_{1}, \ldots, t_{n}, \ldots\right\}, \alpha_{n+1}(\eta)$ satisfying (H1), (H2), (H3) and (H4) and $(\lambda, \mu)$ satisfying (4.2).

It is easy to see that for (4.1), (2.9) reads

$$
\left\{\begin{array}{r}
\bar{y}_{n+1}=\bar{y}_{n}+h_{n+1} \sum_{i=1}^{s} b_{i}\left(\lambda \bar{Y}_{i}^{n+1}+\mu \bar{Y}_{i}^{n-m+1}\right), \\
\bar{Y}_{i}^{n+1}=\bar{y}_{n}+\bar{h}_{n+1} \sum_{j=1}^{s} a_{i j}\left(\lambda \bar{Y}_{j}^{n+1}+\mu \bar{Y}_{j}^{n-m+1}\right), \\
i=1,2, \ldots, s .
\end{array}\right.
$$

If we denote $Y_{n}=\left(\bar{y}_{n}, \bar{Y}_{1}^{n}, \bar{Y}_{2}^{n}, \ldots, \bar{Y}_{s}^{n}\right)^{T}$, then (4.3) can be rewritten as

$$
D_{0}^{(n)} Y_{n+1}=D_{1}^{(n)} Y_{n}+D_{2}^{(n)} Y_{n-m+1}
$$

where $\lambda_{n}=\lambda h_{n}, \bar{\lambda}_{n}=\lambda \bar{h}_{n}, \mu_{n}=\mu h_{n}, \bar{\mu}_{n}=\mu \bar{h}_{n}, e=(1,1, \ldots, 1)^{T} \in \mathbb{C}^{s}$ and

$$
D_{0}^{(n)}=\left(\begin{array}{cc}
1 & -\lambda_{n+1} b^{T} \\
0 & I-\bar{\lambda}_{n+1} A
\end{array}\right), \quad D_{1}^{(n)}=\left(\begin{array}{cc}
1 & 0 \\
e & 0
\end{array}\right), \quad D_{2}^{(n)}=\left(\begin{array}{cc}
0 & \mu_{n+1} b^{T} \\
0 & \bar{\mu}_{n+1} A
\end{array}\right),
$$

which is equivalent to

$$
Y_{n+1}=G_{1}^{(n)} Y_{n}+G_{2}^{(n)} Y_{n-m+1},
$$

with

where

$$
G_{1}^{(n)}=\left(\begin{array}{cc}
\bar{r}_{n+1} & 0 \\
\gamma_{n+1}^{(1)} & 0
\end{array}\right), \quad G_{2}^{(n)}=\left(\begin{array}{cc}
0 & \gamma_{n+1}^{(2)} \\
0 & \gamma_{n+1}^{(3)}
\end{array}\right)
$$

$$
\begin{array}{ll}
\bar{r}_{n+1}=1+\lambda_{n+1} b^{T}\left(I-\bar{\lambda}_{n+1} A\right)^{-1} e, & \gamma_{n+1}^{(1)}=\left(I-\bar{\lambda}_{n+1} A\right)^{-1} e, \\
\gamma_{n+1}^{(2)}=\mu_{n+1} b^{T}\left(I-\bar{\lambda}_{n+1} A\right)^{-1}, & \gamma_{n+1}^{(3)}=\bar{\mu}_{n+1}\left(I-\bar{\lambda}_{n+1} A\right)^{-1} A .
\end{array}
$$

Let $Z_{n}=\left(Y_{n}^{T}, Y_{n-1}^{T}, \ldots, Y_{n-m+1}^{T}\right)^{T}$. Then

$$
Z_{n+1}=Q_{n} Z_{n}
$$

where

$$
Q_{n}=\left(\begin{array}{cccccc}
G_{1}^{(n)} & 0 & \cdots & 0 & 0 & G_{2}^{(n)} \\
I & 0 & \cdots & 0 & 0 & 0 \\
0 & I & \cdots & 0 & 0 & 0 \\
\cdots & & \cdots & & & \cdots \\
0 & 0 & \cdots & I & 0 & 0 \\
0 & 0 & \cdots & 0 & I & 0
\end{array}\right)_{m(s+1)} .
$$


4.1. $H_{\alpha}$-stability with regular $A$. If we denote

$$
\begin{aligned}
\bar{r}_{\infty} & =\lim _{n \rightarrow \infty} \bar{r}_{n+1}, & \gamma^{(1)} & =\lim _{n \rightarrow \infty} \gamma_{n+1}^{(1)}, \\
\gamma^{(2)} & =\lim _{n \rightarrow \infty} \gamma_{n+1}^{(2)}, & \gamma^{(3)} & =\lim _{n \rightarrow \infty} \gamma_{n+1}^{(3)},
\end{aligned}
$$

then from (2.8), (H3) and the regularity of $A$, we have

$$
\begin{aligned}
& \bar{r}_{\infty}=1-\frac{1}{1+\alpha(H)} b^{T} A^{-1} e, \quad \gamma^{(1)}=0, \\
& \gamma^{(2)}=-\frac{1}{1+\alpha(H)} \frac{\mu}{\lambda} b^{T} A^{-1}, \quad \gamma^{(3)}=-\frac{\mu}{\lambda} I_{s} .
\end{aligned}
$$

Let

$$
G_{1}=\lim _{n \rightarrow \infty} G_{1}^{(n)}, \quad G_{2}=\lim _{n \rightarrow \infty} G_{2}^{(n)}, \quad Q=\lim _{n \rightarrow \infty} Q_{n}
$$

then

$$
\begin{aligned}
& G_{1}=\left(\begin{array}{cc}
\bar{r}_{\infty} & 0 \\
\gamma^{(1)} & 0
\end{array}\right), \quad G_{2}=\left(\begin{array}{cc}
0 & \gamma^{(2)} \\
0 & \gamma^{(3)}
\end{array}\right), \\
& Q=\left(\begin{array}{cccccc}
G_{1} & 0 & \cdots & 0 & 0 & G_{2} \\
I & 0 & \cdots & 0 & 0 & 0 \\
0 & I & \cdots & 0 & 0 & 0 \\
\cdots & & \cdots & & & \cdots \\
0 & 0 & \cdots & I & 0 & 0 \\
0 & 0 & \cdots & 0 & I & 0
\end{array}\right)_{m(s+1)}
\end{aligned}
$$

It is easily seen that

$$
\begin{aligned}
& \operatorname{det}\left[\xi I_{m(s+1)}-Q\right]=\operatorname{det}\left[\xi^{m} I_{s+1}-\xi^{m-1} G_{1}-G_{2}\right] \\
& \quad=\operatorname{det}\left[\begin{array}{cc}
\left(\xi-\left(1-\frac{1}{1+\alpha(H)} b^{T} A^{-1} e\right)\right) \xi^{m-1} & \frac{1}{1+\alpha(H)} \frac{\mu}{\lambda} b^{T} A^{-1} \\
0 & \left(\xi^{m}+\frac{\mu}{\lambda}\right) I_{s}
\end{array}\right] \\
& \quad=\xi^{m-1}\left(\xi-\left(1-\frac{1}{1+\alpha(H)} b^{T} A^{-1} e\right)\right)\left(\xi^{m}+\frac{\mu}{\lambda}\right)^{s}
\end{aligned}
$$

and

$$
\bar{r}_{\infty} \text { is an eigenvalue of } Q \text { with the algebraic multiplicity } 1 .
$$

Lemma 4.2. For any given mesh $H$, if $(\mathrm{H} 2)$ and $(\mathrm{H} 3)$ hold, then there is a constant $C>0$ such that

$$
\left\|G_{1}^{(k m+l)}-G_{1}\right\|_{1}+\left\|G_{2}^{(k m+l)}-G_{2}\right\|_{1} \leq C q^{k}, \quad k \geq 0, \quad 0 \leq l \leq m-1 .
$$

Proof. In view of (2.8) and (H2), we can see that there exist constants $M_{1}$ and $M_{2}$ such that for all $n=k m+l, k \geq 0$ and $0 \leq l \leq m-1$,

$$
\left|\frac{b^{T} A^{-2}\left(I-\bar{\lambda}_{n}^{-1} A^{-1}\right)^{-1} e}{\lambda\left(1+\alpha_{n}\left(h_{n}\right)\right)^{2}}\right| \leq M_{1}
$$

and

$$
\left|\frac{b^{T} A^{-1} e}{\left(1+\alpha_{n}\left(h_{n}\right)\right)(1+\alpha(H))}\right| \leq M_{2}
$$


Hence

$$
\begin{aligned}
\mid \bar{r}_{n}- & \bar{r}_{\infty}|=| \lambda_{n} b^{T}\left(I-\bar{\lambda}_{n} A\right)^{-1} e+\frac{1}{1+\alpha(H)} b^{T} A^{-1} e \mid \\
& =\left|b^{T} A^{-1}\left(\frac{1}{1+\alpha(H)} I-\frac{1}{1+\alpha_{n}\left(h_{n}\right)}\left(I-\bar{\lambda}_{n}^{-1} A^{-1}\right)^{-1}\right) e\right| \\
& =\mid b^{T} A^{-1}\left(\left(\frac{1}{1+\alpha(H)}-\frac{1}{1+\alpha_{n}\left(h_{n}\right)}\right) I\right. \\
& \left.\quad+\frac{1}{1+\alpha_{n}\left(h_{n}\right)} I-\frac{1}{1+\alpha_{n}\left(h_{n}\right)}\left(I-\bar{\lambda}_{n}^{-1} A^{-1}\right)^{-1}\right) e \mid \\
& \leq \frac{1}{h_{n}} M_{1}+\left|\alpha_{n}\left(h_{n}\right)-\alpha(H)\right| M_{2} .
\end{aligned}
$$

Therefore it follows from (2.8) and (H3) that

$$
\left|\bar{r}_{k m+l}-\bar{r}_{\infty}\right| \leq q^{k}\left(\frac{1}{h_{l}} M_{1}+\left|\alpha_{l}\left(h_{l}\right)-\alpha(H)\right| M_{2}\right) \leq C_{0} q^{k},
$$

where

$$
C_{0}=\max _{0 \leq l \leq m-1}\left\{\frac{1}{h_{l}} M_{1}+\left|\alpha_{l}\left(h_{l}\right)-\alpha(H)\right| M_{2}\right\} .
$$

In a similar way, there exist constants $C_{i}, i=1,2,3$, such that

$$
\left\|\gamma_{k m+l}^{(i)}-\gamma^{(i)}\right\|_{1} \leq C_{i} q^{k}, \quad k \geq 0, \quad 0 \leq l \leq m-1
$$

Consequently, for all $n=k m+l, k \geq 0$ and $0 \leq l \leq m-1$,

$$
\begin{aligned}
& \left\|G_{1}^{(k m+l)}-G_{1}\right\|_{1}+\left\|G_{2}^{(k m+l)}-G_{2}\right\|_{1} \\
& \quad \leq\left|\bar{r}_{k m+l}-\bar{r}_{\infty}\right|+\sum_{i=1}^{3}\left\|\gamma_{k m+l}^{(i)}-\gamma^{(i)}\right\|_{1} \leq C q^{k},
\end{aligned}
$$

where $C=C_{0}+C_{1}+C_{2}+C_{3}$.

As a result, $\sum_{n=0}^{\infty}\left\|Q_{n}-Q\right\|_{1}<\infty$, which together with (4.6) implies from Theorem 3.3 that

Theorem 4.3. Assume that the matrix $A$ is regular. Then method (2.7) is $H_{\alpha^{-}}$ stable if and only if

$$
\left|1-\frac{1}{1+\alpha(H)} b^{T} A^{-1} e\right|<1 .
$$

Corollary 4.4. The Radau IA, Radau IIA and Lobatto IIIC methods are $H_{\alpha}$-stable.

Corollary 4.5. The Gauss-Legendre methods are $H_{\alpha}$-stable if and only if $s$ is odd.

Corollary 4.6. The one-leg $\theta$-method is $H_{\alpha}$-stable if and only if $\frac{1}{2} \leq \theta \leq 1$.

Proof. In fact, if $\theta<\frac{1}{2}$, then by (H4) there are an $\epsilon>0$ and a mesh $H$ such that $\alpha(H)<\epsilon$ and

$$
\left|\bar{r}_{\infty}\right| \geq\left|1-\frac{1}{\theta} \frac{1}{1+\epsilon}\right|>1
$$

which implies that the numerical solution of the method is not asymptotically stable from Theorem 4.3 . 
4.2. $H_{\alpha}$-stability of a stiffly accurate method. Here we assume that $a_{1 j}=0$, $1 \leq j \leq s$, and write

$$
A=\left(\begin{array}{cc}
0 & 0 \\
\bar{a} & \bar{A}
\end{array}\right), \quad \bar{a}=\left(a_{21}, a_{31}, \ldots, a_{s 1}\right)^{T}, \quad \bar{A}=\left(a_{i j}\right)_{i, j=2}^{s} .
$$

We also assume that $\bar{A}$ is nonsingular and the Runge-Kutta method is stiffly accurate, i.e.,

$$
a_{s j}=b_{j}, \quad 1 \leq j \leq s .
$$

The Lobatto IIIA methods and the linear $\theta$-methods are typical examples of such Runge-Kutta methods.

Although our results in this subsection can be obtained in the same way as [19, we will formulate the expressions of $G_{1}, G_{2}, Q$ and $\operatorname{det}\left[\xi I_{m(s+1)}-Q\right]$, directly. Let $\bar{e}=(1,1, \ldots, 1)^{T} \in \mathbb{C}^{s-1}, e_{s-1}=(0,0, \ldots, 0,1)^{T} \in \mathbb{C}^{s-1}$ and $\bar{I}$ be the identity matric in $\mathbb{C}^{s-1}$. Then

$$
\begin{aligned}
\left(I-\bar{\lambda}_{n+1} A\right)^{-1} & =\left(\begin{array}{cc}
0 & 1 \\
\left(\bar{I}-\bar{\lambda}_{n+1} \bar{A}\right)^{-1} \bar{\lambda}_{n+1} \bar{a} & \left(\bar{I}-\bar{\lambda}_{n+1} \bar{A}\right)^{-1}
\end{array}\right), \\
\left(I-\bar{\lambda}_{n+1} A\right)^{-1} e & =\left(\begin{array}{cc}
\left(\bar{I}-\bar{\lambda}_{n+1} \bar{A}\right)^{-1} \bar{\lambda}_{n+1} \bar{a}+\left(\bar{I}-\bar{\lambda}_{n+1} \bar{A}\right)^{-1} \bar{e}
\end{array}\right), \\
b^{T}\left(I-\bar{\lambda}_{n+1} A\right)^{-1} & =\left(\begin{array}{ll}
e_{s-1}^{T}\left(\bar{I}-\bar{\lambda}_{n+1} \bar{A}\right)^{-1} \bar{a} & e_{s-1}^{T} \bar{A}\left(\bar{I}-\bar{\lambda}_{n+1} \bar{A}\right)^{-1}
\end{array}\right),
\end{aligned}
$$

and

$$
\left(I-\bar{\lambda}_{n+1} A\right)^{-1} A=\left(\begin{array}{cc}
0 & 0 \\
\left(\bar{I}-\bar{\lambda}_{n+1} \bar{A}\right)^{-1} \bar{a} & \left(\bar{I}-\bar{\lambda}_{n+1} \bar{A}\right)^{-1} \bar{A}
\end{array}\right) .
$$

Therefore

$$
\begin{aligned}
& G_{1}^{(n)}=\left(\begin{array}{ccc}
1+\lambda_{n+1} e_{s-1}^{T}\left(I_{s-1}-\bar{\lambda}_{n+1} \bar{A}\right)^{-1} \bar{a}+\lambda_{n+1} e_{s-1}^{T} \bar{A}\left(I_{s-1}-\bar{\lambda}_{n+1} \bar{A}\right)^{-1} \bar{e} & 0 & 0 \\
1 & 0 & 0 \\
\left(\bar{I}-\bar{\lambda}_{n+1} \bar{A}\right)^{-1} \bar{\lambda}_{n+1} \bar{a}+\left(\bar{I}-\bar{\lambda}_{n+1} \bar{A}\right)^{-1} \bar{e} & 0 & 0
\end{array}\right), \\
& G_{2}^{(n)}=\left(\begin{array}{ccc}
0 & \mu_{n+1} e_{s-1}^{T}\left(I_{s-1}-\bar{\lambda}_{n+1} \bar{A}\right)^{-1} \bar{a} & \mu_{n+1} e_{s-1}^{T} \bar{A}\left(I_{s-1}-\bar{\lambda}_{n+1} \bar{A}\right)^{-1} \\
0 & 0 & 0 \\
0 & \bar{\mu}_{n+1}\left(\bar{I}-\bar{\lambda}_{n+1} \bar{A}\right)^{-1} \bar{a} & \bar{\mu}_{n+1}\left(\bar{I}-\bar{\lambda}_{n+1} \bar{A}\right)^{-1} \bar{A}
\end{array}\right), \\
& \bar{r}_{\infty}=1-\frac{1}{1+\alpha(H)} e_{s-1}^{T} \bar{A}^{-1} \bar{a}-\frac{1}{1+\alpha(H)}, \quad \gamma^{(1)}=\left(\begin{array}{c}
1 \\
-\bar{A}^{-1} \bar{a}
\end{array}\right), \\
& \gamma^{(2)}=-\frac{1}{1+\alpha(H)} \frac{\mu}{\lambda}\left(\begin{array}{ll}
e_{s-1}^{T} \bar{A}^{-1} \bar{a} & e_{s-1}^{T}
\end{array}\right), \quad \gamma^{(3)}=-\frac{\mu}{\lambda}\left(\begin{array}{cc}
0 & 0 \\
\bar{A}^{-1} \bar{a} & \bar{I}
\end{array}\right),
\end{aligned}
$$

and

$$
\begin{aligned}
G_{1} & =\left(\begin{array}{ccc}
\bar{r}_{\infty} & 0 & 0 \\
1 & 0 & 0 \\
-\bar{A}^{-1} \bar{a} & 0 & 0
\end{array}\right), \\
G_{2} & =\left(\begin{array}{ccc}
0 & -\frac{1}{1+\alpha(H)} \frac{\mu}{\lambda} e_{s-1}^{T} \bar{A}^{-1} \bar{a} & -\frac{1}{1+\alpha(H)} \frac{\mu}{\lambda} e_{s-1}^{T} \\
0 & 0 & 0 \\
0 & -\frac{\mu}{\lambda} \bar{A}^{-1} \bar{a} & -\frac{\mu}{\lambda} \bar{I}
\end{array}\right),
\end{aligned}
$$

hence

$$
\operatorname{det}\left[\xi^{m} I_{s+1}-\xi^{m-1} G_{1}-G_{2}\right]=\xi^{2 m-1}\left(\xi-\bar{r}_{\infty}\right)\left(\xi^{m}+\frac{\mu}{\lambda}\right)^{s-1}
$$


Consequently, it is easy to see from the discussion in Section 4.1 that the conditions in Theorem 3.3 are satisfied.

Theorem 4.7. Assume that the Runge-Kutta method $(A, b, c)$ is stiffly accurate and the matrix $\bar{A}$ is regular. Then method (2.7) is $H_{\alpha}$-stable if and only if

$$
\left|\bar{r}_{\infty}\right|<1 \text {. }
$$

If $\alpha(H)=0$, then $\bar{r}_{\infty}=\lim _{z \rightarrow \infty} r(z)$, where $r(z)=1+z b^{T}(I-z A)^{-1} e$ is the stability function of the Runge-Kutta method. So we have

Lemma 4.8. If the Runge-Kutta method is stiffly accurate and $\bar{A}$ is regular, then

$$
\lim _{z \rightarrow \infty} r(z)=-e_{s-1}^{T} \bar{A}^{-1} \bar{a}
$$

where $e_{s-1}=(0,0, \ldots, 0,1)^{T} \in \mathbb{C}^{s-1}$.

From Theorem 4.7 and Lemma 4.8 we have

Corollary 4.9. The Lobatto IIIA methods are $H_{\alpha}$-stable if and only if $s$ is even.

Corollary 4.10. The linear $\theta$-method is $H_{\alpha}$-stable if and only if $\frac{1}{2} \leq \theta \leq 1$.

4.3. $H_{\alpha}$-stability of the Lobatto IIIB methods. In this subsection we will investigate the $H_{\alpha}$-stability of the Lobatto IIIB methods. For the Lobatto IIIB, we have

$$
\begin{aligned}
& a_{i 1}=b_{1}, \quad 1 \leq i \leq s, \\
& A=\left(\begin{array}{ll}
\bar{A} & 0 \\
\bar{a} & 0
\end{array}\right),
\end{aligned}
$$

where $\bar{a}=\left(a_{s 1}, a_{s 2}, \ldots, a_{s, s-1}\right)^{T}, \bar{A}=\left(a_{i j}\right)_{i, j=1}^{s-1}$ is regular.

Lemma 4.11. For the Lobatto IIIB methods, $\bar{a} \bar{A}^{-1} \bar{e}=1$.

Proof. Let $r(z)=1+z b^{T}(I-z A)^{-1} e$ be the stability function of the $s$ stage Lobatto IIIB method. Then

$$
\lim _{z \rightarrow \infty} r(z)= \begin{cases}1, & s \text { is odd } \\ -1, & s \text { is even }\end{cases}
$$

and

$$
\begin{aligned}
b^{T}(I-z A)^{-1} e & =\left(\begin{array}{ll}
\bar{b}^{T} & b_{s}
\end{array}\right)\left(\begin{array}{c}
(\bar{I}-z \bar{A})^{-1} \bar{e} \\
z \bar{a}(\bar{I}-z \bar{A})^{-1} \bar{e}+1
\end{array}\right) \\
& =\bar{b}^{T}(\bar{I}-z \bar{A})^{-1} \bar{e}+z b_{s} \bar{a}(\bar{I}-z \bar{A})^{-1} \bar{e}+b_{s} .
\end{aligned}
$$

Hence $0=\lim _{z \rightarrow \infty} b^{T}(I-z A)^{-1} e=b_{s}\left(-\bar{a} \bar{A}^{-1} \bar{e}+1\right)$, i.e., $\bar{a} \bar{A}^{-1} \bar{e}=1$.

Let $\bar{Y}^{n}=\left(\bar{Y}_{1}^{n}, \bar{Y}_{2}^{n}, \ldots, \bar{Y}_{s-1}^{n}\right)^{T}$ and

$$
P=\left(\begin{array}{ccc}
1 & 0 & 0 \\
0 & \bar{I} & 0 \\
0 & -\bar{a}^{-1} & 1
\end{array}\right) .
$$


Then multiplying (4.4) by $P$ and denoting $\hat{D}_{i}^{(n)}=P D_{i}^{(n)}$ we have

$$
\begin{aligned}
\hat{D}_{0}^{(n)} & =\left(\begin{array}{ccc}
1 & -\lambda_{n+1} \bar{b}^{T} & -\lambda_{n+1} b_{s} \\
0 & \bar{I}-\bar{\lambda}_{n+1} \bar{A} & 0 \\
0 & -\bar{a} \bar{A}^{-1} & 1
\end{array}\right), \hat{D}_{1}^{(n)}=\left(\begin{array}{ccc}
1 & 0 & 0 \\
\bar{e} & 0 & 0 \\
0 & 0 & 0
\end{array}\right), \\
\hat{D}_{2}^{(n)} & =\left(\begin{array}{ccc}
0 & \mu_{n+1} \bar{b}^{T} & \mu_{n+1} b_{s} \\
0 & \bar{\mu}_{n+1} \bar{A} & 0 \\
0 & 0 & 0
\end{array}\right),
\end{aligned}
$$

and (4.4) becomes

$$
\begin{aligned}
\bar{y}_{n+1}-\lambda_{n+1} b^{T}\left(\begin{array}{c}
\bar{Y}^{n+1} \\
\bar{Y}_{s}^{n+1}
\end{array}\right) & =\bar{y}_{n}+\mu_{n+1} b^{T}\left(\begin{array}{c}
\bar{Y}^{n-m+1} \\
\bar{Y}_{s}^{n-m+1}
\end{array}\right), \\
\left(\bar{I}-\bar{\lambda}_{n+1} \bar{A}\right) \bar{Y}^{n+1} & =\bar{e} \bar{y}_{n}+\bar{\mu}_{n+1} \bar{A} \bar{Y}^{n-m+1}, \\
-\bar{a} \bar{A}^{-1} \bar{Y}^{n+1}+\bar{Y}_{s}^{n+1} & =0 .
\end{aligned}
$$

Submitting (4.17) into (4.15) we can obtain

$$
\bar{y}_{n+1}-\lambda_{n+1} \beta^{T} \bar{Y}^{n+1}=\bar{y}_{n}+\mu_{n+1} \beta^{T} \bar{Y}^{n-m+1},
$$

where $\bar{b}=\left(b_{1}, b_{2}, \ldots, b_{s-1}\right)^{T}$ and $\beta^{T}=\bar{b}^{T}+b_{s} \bar{a} \bar{A}^{-1}$. Let $\bar{Y}_{n}=\left(\bar{y}_{n},\left(\bar{Y}^{n}\right)^{T}\right)^{T}$. From (4.16) and (4.18) we have

$$
\bar{D}_{0}^{(n)} \bar{Y}_{n+1}=\bar{D}_{1}^{(n)} \bar{Y}_{n}+\bar{D}_{2}^{(n)} \bar{Y}_{n-m+1}
$$

where

$$
\bar{D}_{0}^{(n)}=\left(\begin{array}{cc}
1 & -\lambda_{n+1} \beta^{T} \\
0 & I-\bar{\lambda}_{n+1} \bar{A}
\end{array}\right), \quad \bar{D}_{1}^{(n)}=\left(\begin{array}{cc}
1 & 0 \\
\bar{e} & 0
\end{array}\right), \quad \bar{D}_{2}^{(n)}=\left(\begin{array}{cc}
0 & \mu_{n+1} \beta^{T} \\
0 & \bar{\mu}_{n+1} \bar{A}
\end{array}\right),
$$

which is equivalent to

$$
\bar{Y}_{n+1}=\bar{G}_{1}^{(n)} \bar{Y}_{n}+\bar{G}_{2}^{(n)} \bar{Y}_{n-m+1},
$$

with

$$
\bar{G}_{1}^{(n)}=\left(\begin{array}{cc}
\bar{R}_{n+1} & 0 \\
\bar{\gamma}_{n+1}^{(1)} & 0
\end{array}\right), \quad \bar{G}_{2}^{(n)}=\left(\begin{array}{cc}
0 & \bar{\gamma}_{n+1}^{(2)} \\
0 & \bar{\gamma}_{n+1}^{(3)}
\end{array}\right)
$$

where

$$
\begin{array}{ll}
\bar{R}_{n+1}=1+\lambda_{n+1} \beta^{T}\left(\bar{I}-\bar{\lambda}_{n+1} \bar{A}\right)^{-1} \bar{e}, & \bar{\gamma}_{n+1}^{(1)}=\left(\bar{I}-\bar{\lambda}_{n+1} \bar{A}\right)^{-1} \bar{e}, \\
\bar{\gamma}_{n+1}^{(2)}=\mu_{n+1} \beta^{T}\left(\bar{I}-\bar{\lambda}_{n+1} \bar{A}\right)^{-1}, & \bar{\gamma}_{n+1}^{(3)}=\bar{\mu}_{n+1}\left(\bar{I}-\bar{\lambda}_{n+1} \bar{A}\right)^{-1} \bar{A} .
\end{array}
$$

By the same argument as in Section 4.1, let $\bar{G}_{i}=\lim _{n \rightarrow \infty} \bar{G}_{i}^{(n)}, i=1,2$. Then

$$
\bar{G}_{1}=\left(\begin{array}{cc}
1-\frac{1}{1+\alpha(H)} \beta^{T} \bar{A}^{-1} \bar{e} & 0 \\
0 & 0
\end{array}\right), \quad \bar{G}_{2}=\left(\begin{array}{cc}
0 & -\frac{1}{1+\alpha(H)} \frac{\mu}{\lambda} \beta^{T} \bar{A}^{-1} \\
0 & -\frac{\mu}{\lambda} \bar{I}
\end{array}\right),
$$

and

$$
\operatorname{det}\left[\xi^{m} I_{s}-\xi^{m-1} \bar{G}_{1}-\bar{G}_{2}\right]=\xi^{s-1}\left(\xi-\bar{R}_{\infty}\right)\left(\xi+\frac{\mu}{\lambda}\right)^{s-1},
$$

where $\bar{R}_{\infty}=1-\frac{1}{1+\alpha(H)} \beta^{T} \bar{A}^{-1} \bar{e}$. It is obvious that the conditions in Theorem 3.3 are satisfied. 
Theorem 4.12. The Lobatto IIIB method $(A, b, c)$ is $H_{\alpha}$-stable if and only if

$$
\left|\bar{R}_{\infty}\right|<1 \text {. }
$$

Lemma 4.13. Let $R(z)=1+z \beta^{T}(I-z \bar{A})^{-1} \bar{e}$ and $r(z)$ be the stability function. Then for the Lobatto IIIB method, we have

(1) $R(z)=r(z)$ for all $z \in \mathbb{C}$;

(2) $R_{\infty}=\lim _{z \rightarrow \infty} R(z)=1-\beta^{T} \bar{A}^{-1} \bar{e}$;

(3) $\beta^{T} \bar{A}^{-1} \bar{e}=2$ when $s$ is even and $\beta^{T} \bar{A}^{-1} \bar{e}=0$ when $s$ is odd.

Proof. In fact, in view of $\bar{a} \bar{A}^{-1} \bar{e}=1$ we have

$$
\begin{aligned}
b^{T}(I-z A)^{-1} e & =\left(\begin{array}{ll}
\bar{b}^{T} & b_{s}
\end{array}\right)\left(\begin{array}{cc}
(\bar{I}-z \bar{A})^{-1} & 0 \\
z \bar{a}(\bar{I}-z \bar{A})^{-1} & \bar{a} \bar{A}^{-1} \bar{e}
\end{array}\right)\left(\begin{array}{c}
\bar{e} \\
1
\end{array}\right) \\
= & \left(\bar{b}^{T}+b_{s} \bar{a} \bar{A}^{-1}\right)(\bar{I}-z \bar{A})^{-1} \bar{e}=\beta^{T}(\bar{I}-z \bar{A})^{-1} \bar{e} .
\end{aligned}
$$

Therefore (1) holds. (2) holds since $\bar{A}$ is regular, which together with (1) implies (3).

From Theorem 4.12 and Lemma 4.13 we have

Corollary 4.14. The Lobatto IIIB methods are $H_{\alpha}$-stable if and only if $s$ is even.

\section{Numerical EXPERIMENTS}

Let the Runge-Kutta method $(A, b, c)$ be of order $p$, let $H=\left\{m ; t_{0}, t_{1}, \ldots\right\}$ be a given mesh, $h_{n+1}=t_{n+1}-t_{n}$ and $h=\min _{1 \leq i \leq m} h_{i}$. We define for $n=k m+l$, $l=1,2, \ldots, m$,

$$
\alpha_{k m+l}(\eta)= \begin{cases}h, & p=1, \\ \left(\frac{\eta}{q^{-k} \delta_{l}}\right)^{p-1}, & p \geq 2,\end{cases}
$$

where $\delta_{l}=\frac{h_{l}}{h}$. In fact

$$
\alpha_{n+1}\left(h_{n+1}\right)= \begin{cases}h^{p-1}, & p \geq 2 \\ h, & p=1 .\end{cases}
$$

Therefore (H1), (H2) (H3) and (H4) hold.

Let $H=\left\{m ; t_{0}, t_{1}, \ldots\right\}$ be a geometric mesh which is defined by

$$
t_{n}=q^{-\frac{n}{m}}, n \geq-m .
$$

Then from (5.1) we have $\bar{h}_{n+1}=h_{n+2}$ for $p=2$.

In Table 1, $a=-1, b=0.5, q=0.5, \bar{h}_{n+1}=h_{n+2}$, we list the absolute errors $(A E)$ and relative errors $(R E)$ at $t=16$ of the one-leg $\theta$-method with geometric mesh and the Ratio of the errors of the case $m=50$ over that of $m=100$. From Table 1, we can see that the one-leg $\theta$-method is of order 2 if $\theta=\frac{1}{2}$ and of order 1 if $\theta=0$.

In Figures 1 and 2. $a=-1, b=0.5, q=0.5, m=10, \bar{h}_{n+1}=h_{n+2}, \theta=\frac{1}{2}$. It can be seen from these figures that the numerical solution $\bar{y}_{n}$ of the modified one-leg $\theta$-method with $\theta=\frac{1}{2}$ tends to zero as $n \rightarrow \infty$, whereas the numerical solution $y_{n}$ does not. 
TABLE 1. The one-leg $\theta$-method with geometric mesh

\begin{tabular}{c|c|c|c|c}
\hline \multirow{2}{*}{} & \multicolumn{2}{|c|}{$\theta=0.5$} & \multicolumn{2}{c}{$\theta=0$} \\
\cline { 2 - 5 } & $A E$ & $R E$ & $A E$ & $R E$ \\
\hline$m=2$ & $1.7927 E-2$ & $2.1150 E-1$ & $3.6256 E-3$ & $4.2774 E-2$ \\
$m=3$ & $1.0905 E-2$ & $1.2865 E-1$ & $6.9657 E-3$ & $8.2179 E-2$ \\
$m=5$ & $5.0172 E-3$ & $5.9191 E-2$ & $4.5034 E-3$ & $5.3131 E-2$ \\
$m=10$ & $1.5092 E-3$ & $1.7806 E-2$ & $2.2610 E-3$ & $2.6674 E-2$ \\
$m=20$ & $4.1444 E-4$ & $4.8894 E-3$ & $1.1321 E-3$ & $1.3356 E-2$ \\
$m=50$ & $7.0197 E-5$ & $8.2817 E-4$ & $4.5316 E-4$ & $5.3463 E-3$ \\
$m=100$ & $1.7888 E-5$ & $2.1104 E-4$ & $2.2663 E-4$ & $2.6738 E-3$ \\
\hline Ratio & 3.9243 & 3.9243 & 1.9996 & 1.9996 \\
\hline \multicolumn{4}{|c}{}
\end{tabular}

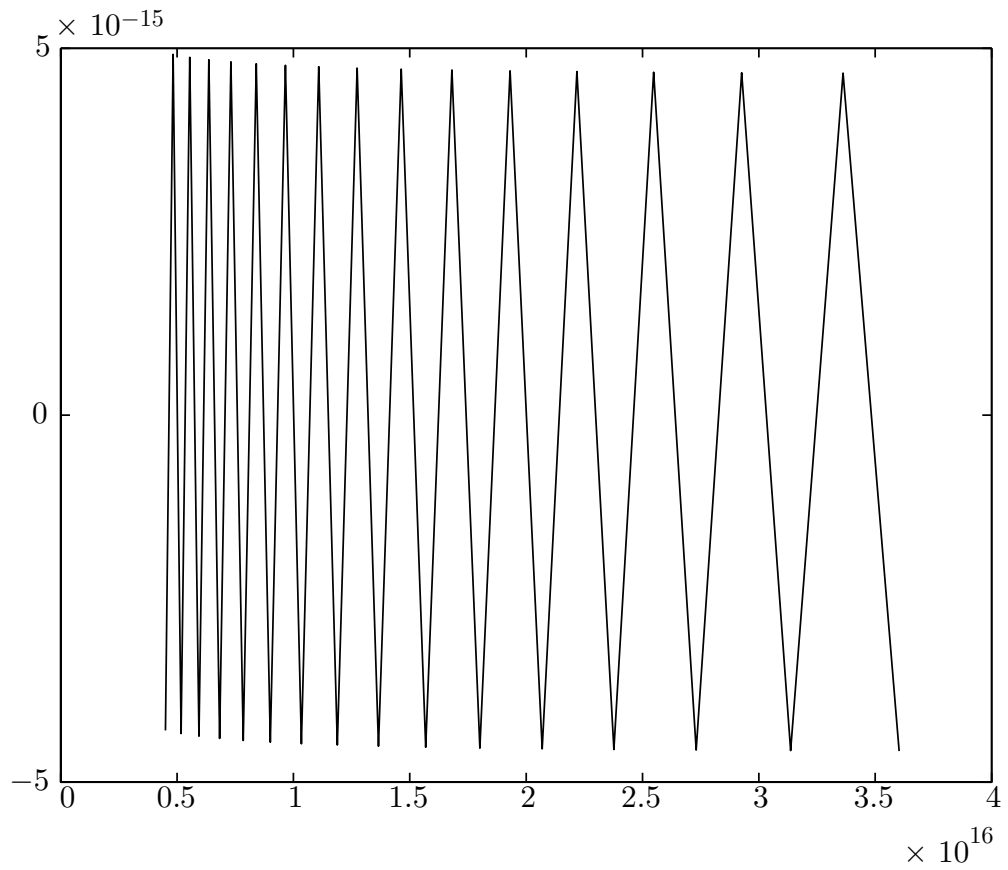

Figure 1. The numerical solution $y_{n}$ of the classical one-leg $\theta$-method

Finally we give some experiments of the Runge-Kutta method with quasi-geometric mesh, which is defined by

$$
t_{n}=T_{k}+(n-k m)\left(\frac{T_{k+1}-T_{k}}{m}\right), k m \leq n<(k+1) m,
$$

where $T_{k}=q^{-k}, k \geq-1$ and $m$ is an integer.

In Table 2, $a=-1, b=0.95, q=0.5, \bar{h}_{n+1}$ defined by (5.1), we list the absolute errors $(A E)$ and relative errors $(R E)$ at $t=16$ of the 3 -Gauss method and 2Lobatto IIIB method with the quasi-geometric mesh and the Ratio of the errors of the case $m=50$ over that of $m=100$. The table shows that the methods defined in this paper can preserve the order of the classical methods. 
TABLE 2. The Runge-Kutta methods with quasi-geometric mesh

\begin{tabular}{c|c|c|c|c}
\hline \multirow{2}{*}{} & \multicolumn{2}{|c|}{ 3-Gauss } & \multicolumn{2}{c}{ 2-Lobatto IIIB } \\
\cline { 2 - 5 } & $A E$ & $R E$ & $A E$ & $R E$ \\
\hline$m=2$ & $3.1521 E-2$ & $3.8295 E-2$ & $2.7342 E-1$ & $3.3217 E-1$ \\
$m=3$ & $3.1566 E-3$ & $3.8349 E-3$ & $1.6546 E-1$ & $2.0102 E-1$ \\
$m=5$ & $1.5897 E-4$ & $1.9314 E-4$ & $7.6110 E-2$ & $9.2465 E-2$ \\
$m=10$ & $2.5963 E-6$ & $3.1542 E-6$ & $2.2717 E-2$ & $2.7599 E-2$ \\
$m=20$ & $4.1279 E-8$ & $5.0149 E-8$ & $6.1802 E-3$ & $7.5083 E-3$ \\
$m=50$ & $1.7057 E-10$ & $2.0722 E-10$ & $1.0383 E-3$ & $1.2615 E-3$ \\
$m=100$ & $2.6728 E-12$ & $3.2471 E-12$ & $2.6375 E-4$ & $3.2042 E-4$ \\
\hline Ratio & 63.818 & 63.818 & 3.9369 & 3.9369 \\
\hline
\end{tabular}

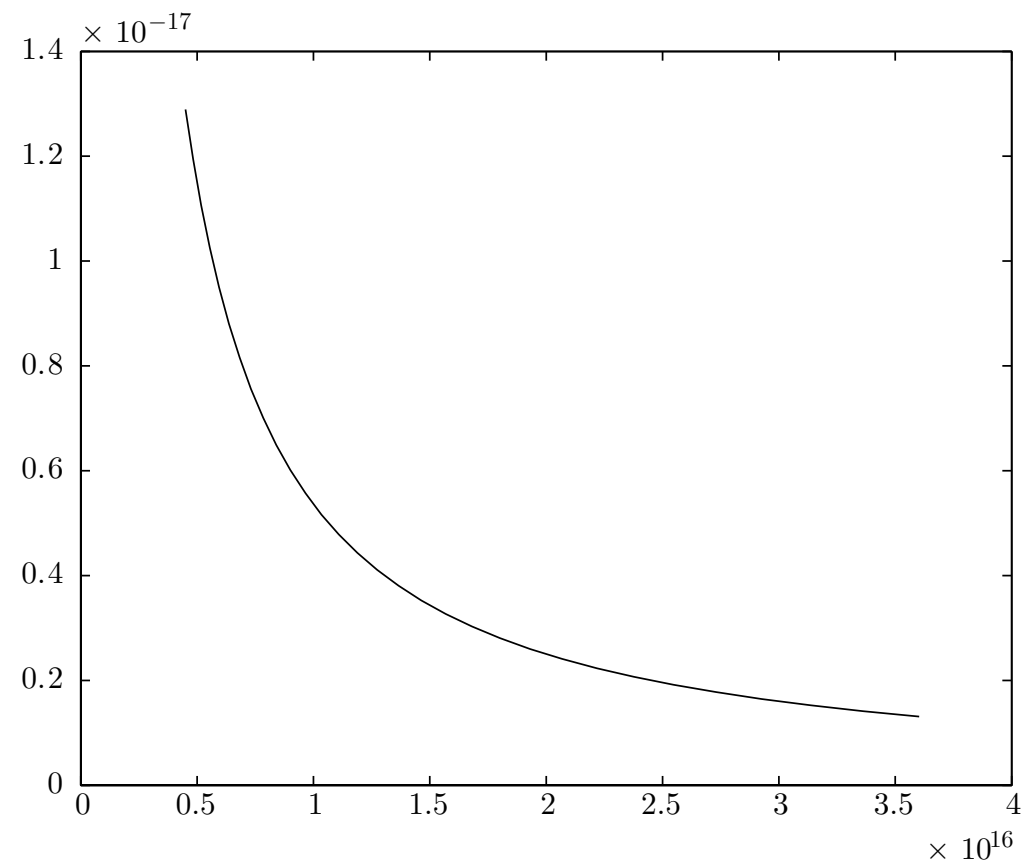

Figure 2. The numerical solution $\bar{y}_{n}$ of the modified one-leg $\theta$-method

\section{REFERENCES}

1. A. Bellen, Preservation of superconvergence in the numerical integration of delay differential with proportional delay, IMA Journal of Numerical Analysis, 22 (2002), 529-536. MR.1936518 (2003j:65060)

2. A. Bellen, N. Guglielmi and L. Torelli, Asymptotic stability properties of $\theta$-methods for the pantograph equation, Appl. Numer. Math., 24 (1997), pp. 279-293. MR1464729 (98f:34093)

3. A. Bellen and M. Zennaro, Numerical methods for delay differential equations, Numerical Mathematics and Scientific Computation, The Clarendon Press, Oxford University Press, New York, 2003. MR1997488(2004i:65001)

4. J. Carr and J. Dyson, The functional differential equation $y^{\prime}(x)=a y(\lambda x)+b y(x)$, Proc. Roy. Soc. Edinburgh. Sect. A, 13 (1975), pp. 165-174. MR 0442421 (56:803)

5. G.A. Derfel, Kato problem for functional equations and difference Schrödinger operators, Operator Theory, 46 (1990), pp. 319-321. MR1124676 (92g:34093) 
6. S.N. Elaydi, An Introduction to Difference Equations, Springer-Verlag, New York, 1999. MR 1711587 (2001g:39001)

7. L. Fox, D.F. Mayers, J.R. Ockendon and A.B. Tayler, On a functional differential equation, J. Inst. Math. Appl., 8 (1971), pp. 271-307. MR0301330 (46:488)

8. R.A. Horn and C.R. Johnson, Matrix Analysis, Cambridge University Press. Cambridge, 1985. MR 0832183 (87e:15001)

9. A. Iserles, On the generalized pantograph functional-differential equation, European J. Appl. Math., 4 (1993), pp. 1-38. MR.1208418 (94f:34127)

10. A. Iserles, Numerical analysis of delay differential equations with variable delays, Ann. Numer. Math., 1 (1994), pp. 133-152. MR.1340650 (96d:65130)

11. A. Iserles and J. Terjeki, Stability and asymptotical stablity of functional-differential equations, J. London. Math. Soc., 51 (2) (1995), pp. 559-572. MR1332892 (96c:34171)

12. A. Iserles, Exact and discretized stability of the pantograph equation, Appl. Numer. Math., 24 (1997), pp. 295-308. MR1464730 (98j:34130)

13. T. Kato and J.B. Mcleod, The functional-differential equation $y^{\prime}(x)=a y(\lambda x)+b y(x)$, Bull. Amer. Math. Soc., 77 (1971), pp. 719-731. MR0283338 (44:570)

14. T. Koto, Stability of Runge-Kutta methods for the generalized pantograph equation, Numer. Math, 84 (1999), pp. 870-884. MR1730016 (2000k:65115)

15. Y. Liu, Stablity of $\theta$-methods for neutral functional-differential equations, Numer.Math., 70 (1995), pp. 473-485. MR1337227 (96d:65127)

16. Y. Liu, On the $\theta$-method for delay differential equations with infinite lag, J. Comput. Appl. Math., 71 (1996), pp. 177-190. MR.1399890 (97c:65136)

17. Y. Liu, Numerical investigation of the pantograph equation, Appl. Numer. Math., 24 (1997), pp. 309-317. MR.1464731 (98j:34131)

18. J.R. Ockendon and A.B. Tayler, The dynamics of a current collection system for an electric locomotive, Proc. Roy. Soc. Edinburg Sect. A, 322 (1971), pp. 447-468.

19. Y. Xu and M.Z. Liu, $\mathcal{H}$-stability of Runge-Kutta methods with general variable stepsize for pantograph equation, Appl. Math. Comput., 148 (2004), pp. 881-892. MR2024551 (2004j:65091)

Department of Mathematics, Harbin Institute of Technology, Harbin 150001, PeoPLE's RePublic of China

E-mail address: mzliu@hope.hit.edu.cn

Department of Mathematics, Harbin Institute of Technology, Harbin 150001, PeoPLE's Republic of China

Department of Mathematics, Harbin Institute of Technology, Harbin 150001, PeoPLE'S REPUBLic OF ChinA 